\title{
Fisioterapia no equilíbrio de pacientes pós Acidente Vascular Encefálico com enfoque na melhora da marcha: revisão sistemática
}

1.

Physiotherapy in the balance of patients

of post-stroke with a focus on gait

FisiSenectus . Unochapecó Ano 6, n. 2 - Jul/Dez. 2018 improvement: systematic review

Jossinelma Camargo Gomes. jossinelma@hotmail.com

Fisioterapeuta, graduada na Universidade Estadual do Centro-Oeste do Paraná (Unicentro-PR). Discente do Programa de Pós-Graduação em Biociências e Saúde pela Universidade Estadual do Oeste do Paraná (UNIOESTE).

Gabriela Antonelli. gabrielaantonellibss@gmail.com

Fisioterapeuta, graduada na Universidade Estadual do Centro-Oeste do Paraná (Unicentro-PR). Residente em Terapia Intensiva pela Universidade Estadual do Oeste do Paraná (UNIOESTE).

Jackeline Siqueira Dalzoto. jackeline_dalzoto@hotmail.com

Fisioterapeuta, graduada na Universidade Estadual do Centro-Oeste do Paraná (Unicentro-PR). Residente em Saúde do Idoso pela Universidade Estadual de Ponta Grossa (UEPG).

Gabrielly Alves Pereira. fisiogabi@outlook.com.br

Fisioterapeuta, graduada na Universidade Estadual do Centro-Oeste do Paraná (Unicentro-PR).

Ana Carolina Dorigoni Bini. ana.carolina.db@hotmail.com

Fisioterapeuta, docente do Departamento de Fisioterapia da Universidade Estadual do Centro-Oeste do

Paraná (UNICENTRO-PR). Doutoranda no Programa de Ciências Farmacêuticas da UNICENTRO.

\section{Resumo}

Introdução: Em geral, pacientes acometidos por Acidente Vascular Encefálico (AVE) apresentam déficit no equilíbrio, o que traz diversas complicações, como insegurança, risco de quedas aumentado e alterações na marcha, afetando negativamente a funcionalidade do paciente. Assim, é fundamental a recuperação do equilíbrio através da reabilitação motora, o que repercutirá na marcha e funcionalidade do paciente. Objetivo: O objetivo do estudo foi identificar as técnicas fisioterapêuticas realizadas para melhorar o equilíbrio estático e dinâmico em pacientes pós-AVE. Materiais e métodos: foi realizada uma busca de artigos publicados entre 2013 e 2017 em três bases de dados - PubMed, Scielo e Lilacs. Resultados: A busca resultou em 6.771 artigos (6.674 PubMed, 39 Scielo e 58 Lilacs); destes, foram exclusos 6.736 pelo título e 28 pelo resumo. Foram inclusos 5 artigos na revisão. Conclusão: 0 fortalecimento muscular e o treino de equilíbrio associados à realidade virtual melhoram o equilíbrio em pacientes pós-AVE.

\section{Palavras-chave}

Marcha; Realidade Virtual; Funcionalidade.

\section{Fisißenectus}




\begin{abstract}
Introduction: In general, patients with stroke present a deficit in balance, which brings complications such insecurity, risk of falls increased and altering in gait, affecting negatively the patient functionality Thus, balance recovery through motor rehabilitation is fundamental, which will affect the gait and functionality of the patient. Objective: The objective this study was to identify the physiotherapeutic techniques to improve the static and dynamic balance in post-stroke patients. Materials and methods: was searched the articles published between 2013 and 2017 in three databases - PubMed, Scielo and Lilacs. Results: The search resulted in 6.771 articles (6.674 Scielo, 39 Scielo and 58 Lilacs), of which 6.736 were excluded by the title and 28 by the abstract. Were included 5 articles this is study. Conclusion: The muscle strengthening and balance training associated with virtual reality for improve do balance in post-stroke patients.
\end{abstract}

\title{
Keywords
}

Gait; Virtual reality; Functionality.

\section{Introdução}

$\infty \infty \infty \times \infty \times \infty \times \infty \times \infty \times \infty \times \infty \times \infty \times \infty \times \infty \times \infty \times \infty$

O Acidente Vascular Encefálico (AVE) pode trazer diversos prejuízos ao indivíduo, como distúrbio do equilíbrio e de mobilidade, em decorrência da paralisia e fraqueza muscular dos membros inferiores $^{1}$.

Distúrbios relacionados ao controle postural são comuns em pacientes hemiparéticos com sequelas de $\mathrm{AVE}$, esses distúrbios podem trazer insegurança, risco de quedas e diferentes níveis de dependência funcional².

Segundo Tanaka e Scheicher ${ }^{3}$, existe presença de risco de quedas aumentando em indivíduos pós-AVE e tende a permanecer na fase crônica. Assim, enfatiza-se a importância da restauração do equilíbrio de pacientes pós-AVE.

As alterações na resposta muscular e os movimentos compensatórios prejudicam as estratégias de equilíbrio, o que dificulta o controle e a execução da marcha em pacientes com hemiparesia. Assim, a avaliação do equilíbrio é fundamental em programas de reabilitação motora que visam a melhorar a funcionalidade dos indivíduos ${ }^{4}$.

0 equilíbrio diminuído pode ser devido as alterações cognitivas, diminuição da força muscular, amplitude de movimento limitada, tônus muscular anormal, movimentos descoordenados ou alterações na integração sensorial ${ }^{5}$. Assim, o equilíbrio é um requisito fundamental para restabelecer a marcha ${ }^{6}$.

0 presente estudo apresenta-se como relevante, já que a grande maioria de pacientes pós-AVE apresentam algum grau de déficit de equilíbrio, o que pode afetar a terapia, se não for trabalhado ou trazer malefícios para o paciente, como quedas e insegurança para a realização das atividades de vida diária. Se o equilíbrio for incluso no plano de tratamento, haverá benefícios ao paciente repercutindo de forma positiva para outros quesitos afetados, por exemplo, a marcha e a independência.

O objetivo deste estudo foi identificar as técnicas fisioterapêuticas realizadas para melhorar o equilíbrio estático e dinâmico em pacientes pós-AVE.

\section{Materiais e Métodos}

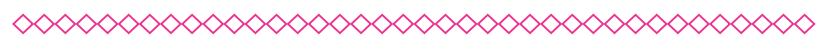

Foi realizada uma busca de artigos publicados entre 2013 e 2017 em três bases de dados: PubMed, Scielo e Lilacs. A seleção dos artigos ocorreu no mês de agosto de 2017 e a leitura, nos meses de setembro e outubro de 2017.

Foram utilizados os descritores nos idiomas português e inglês, respectivamente: acidente vascular cerebral, acidente vascular encefálico, equilíbrio postural no acidente vascular encefálico, fisioterapia no acidente vascular encefálico, stroke, 
postural balance in stroke, physical therapay in stroke.

Foram inclusos ensaios clínicos randomizados (ECR) que abordavam técnicas fisioterapêuticas para melhorar o equilíbrio dinâmico e estático em pacientes deambulantes pós-AVE, em fase ambulatorial, aguda, subaguda ou crônica. Foram selecionadas as técnicas que podem ser trabalhadas na clínica ou no domicílio.

Foram exclusos os estudos que utilizavam tecnologia de alto custo, os quais são inacessíveis à maioria das clínicas, e aqueles que abordavam terapias não convencionais que necessitam de formações/especializações.

\section{Resultados}

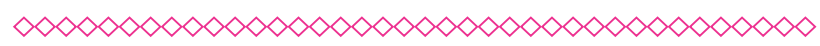

A busca resultou em 6.771 resultados $(6.674$ PubMed, 39 Scielo e 58 Lilacs). Destes, foram exclusos 6.736 pelo título, e selecionados pelo resumo 28 artigos. Dos 28 artigos selecionados para a leitura na íntegra, 5 foram inclusos na revisão. No Fluxograma 1, está disposto o resumo dos procedimentos anteriormente citados.

Na Tabela 1, consta a descrição dos artigos selecionados para a presente pesquisa. De forma breve, são apresentados os autores, a amostra dos estudos, a abordagem fisioterapêutica, os instrumentos/as avaliações utilizadas e os resultados encontrados nesses trabalhos.

\section{Discussão}

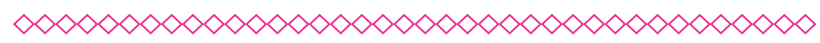

Son, Park, Lee ${ }^{7}$ e Al-Jarrah et al. ${ }^{8}$ observaram melhora do equilíbrio estático e dinâmico em pacientes com AVE através de exercícios de fortalecimento muscular. Enquanto, Lim, Kim e Lee ${ }^{9}$ observaram melhora no equilíbrio estático e dinâmico em pacientes com AVE após 24 sessões de exercícios de Pilates.

Haruyama, Kawakami e Otsuka ${ }^{10}$ realizam um estudo com 32 pacientes com AVE, no qual o grupo experimental realizou 400 minutos de treinamento de estabilidade do tronco e o grupo controle recebeu apenas programas convencionais. 0 treino de estabilidade melhorou a função do tronco, o equilíbrio e a mobilidade dos pacientes.

No entanto, são escassos os estudos sobre treinamento de tronco em pacientes com AVE, assim, não estão bem esclarecidos na literatura quais tipos de treinamento de tronco são mais úteis para melhorar o desempenho do tronco em pacientes com AVE $^{9}$. Sendo que, a estabilização do tronco é geralmente empregado juntamente ao treinamento de força e resistência muscular ${ }^{10}$.

Em um estudo, realizou-se um treinamento em grupo com exercícios de fortalecimento muscular, equilíbrio estático e dinâmico, coordenação motora fina e grosseira e alongamentos. Houve melhora na funcionalidade e no equilíbrio dos pacientes com a conduta proposta ${ }^{4}$.

Com base na leitura foi observado que os autores, em sua maioria, indicam a inclusão de fortalecimento muscular junto ao treino de equilíbrio e não apenas o treino de equilíbrio propriamente dito para a melhora do equilíbrio em pacientes pós-AVE.

A vantagem da terapia por realidade virtual se baseia na possibilidade de trabalhar tarefas orientadas para objetivos, podendo repetir várias vezes a tarefa ${ }^{12}$. Em um estudo com dez indivíduos com AVE unilateral crônico, realizaram oito sessões de cinesioterapia, realidade virtual e exercícios de transferência de aprendizagem. O programa de reabilitação convencional combinado à realidade virtual melhorou o equilíbrio e a funcionalidade dos pacientes pós-AVE ${ }^{13}$.

Choi et al. ${ }^{14}$ avaliaram 50 pacientes com AVE em fase subaguda. Destes, 25 fizeram um treinamento com Wii Fit e terapia convencional para equilíbrio e 25 realizaram somente terapia de equilíbrio convencional. 0 grupo que participou do treino Wii Fit obteve melhoras mais acentuadas do equilíbrio do que o grupo de treino de equilíbrio convencional.

Costa et al. ${ }^{15}$ verificaram a influência imediata do enfaixamento em oito participantes no equilíbrio estático e distribuição da pressão plantar em pacientes com hemiparesia pós-AVE. 0 tratamento proposto não melhorou o equilíbrio estático e a distribuição da pressão plantar, porém, houve 
relato dos pacientes de maior segurança durante a marcha após a terapia.

Filho e Albuquerque ${ }^{16}$ investigaram os efeitos da restrição do membro superior não parético sobre o equilíbrio e a mobilidade funcional em pacientes hemiparéticos crônico e verificaram que o uso da terapia de restrição por seis horas diárias influenciou positivamente na velocidade da marcha.

Park et al. ${ }^{17}$ trabalharam com 34 pacientes ambulatoriais pós-AVE, os quais foram divididos em grupo TENS e placebo. O grupo TENS realizou exercício terapêutico com TENS, enquanto o grupo TENS placebo (não estimulado) realizou exercício terapêutico com TENS placebo. A velocidade de oscilação ântero-posterior e médio-lateral mostraram diferenças entre os grupos. O exercício terapêutico associado ao TENS reduziu a espasticidade e melhorou o equilíbrio, a marcha e a funcionalidade dos pacientes.

A escolha da terapia mais adequada ao paciente dependerá de uma avaliação fisioterapêutica minuciosa, aplicando-se as escalas específicas para avaliar o equilíbrio e verificar o déficit de equilíbrio e quantificar ou qualificar a melhora do paciente. Além disso, o fisioterapeuta pode tornar o tratamento mais dinâmico e atrativo com atividades lúdicas, trabalhando o equilíbrio de diversas formas. 0 fisioterapeuta também deve associar várias técnicas e não apenas trabalhar o equilíbrio propriamente dito. Assim, enfatiza-se a importância de trabalhar o equilíbrio em pacientes pós-AVE para melhorar o equilíbrio, a mobilidade, a marcha e a segurança do paciente ao deambular.

Diversas alternativas para melhorar o equilíbrio são abordadas na literatura como o uso de TENS, terapia do espelho, Bobath, Pilates, plataforma vibratória, hidroterapia, estimulação transcraniana, dentre outras. Essas terapias alternativas não foram descritas no trabalho, pois não se enquadravam ao objetivo do estudo.

\section{Conclusão}

$\infty \times \infty \times \infty \times \infty \times \infty \times \infty \times \infty \times \infty \times \infty \times \infty \times \infty \times \infty \infty \infty$

Dentre as técnicas utilizadas para melhorar o equilíbrio em pacientes pós-AVE, destacam-se como eficazes, a terapia convencional de equilíbrio associada ao fortalecimento muscular e o treinamento por realidade virtual.

Dentre os limites do estudo, ressalta-se a falta de informação nos artigos. Existem relatos de quais atividades fisioterapêuticas foram empregadas nos pacientes, porém, não há a descrição dessas atividades para que seja possível a reprodução exata das técnicas. Outra limitação é em relação à realidade virtual, visto que os autores apenas usam o termo "realidade virtual", não destacando quais foram os jogos utilizados com os pacientes.

\section{Referências}

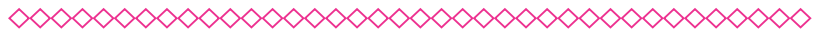

1. Jorgensen HS, Nakayama H, Raaschou HO, Olsen TS. Recovery of walking function in stroke patients: the Copenhagen Stroke Study. Arch Phys Med Rehabil. 1995; 76:27-32.

2. Santos FMK, Mendes FV, Woellner SS, Borges JNG, Soares AV. Use of visual feedback for balance training in hemiparetic Stroke patients. Fisioter. mov. 2015;28(2):241-49.

3. Tanaka AFD, Scheicher ME. Relação entre depressão e desequilíbrio postural em idosos que sofreram acidente vascular encefálico. Fisioter. mov. 2013;26(2):315-20.

4. Amaral-Felipe KM, Yamada PA, Marques AEZS, Pedroni CR, Navega FRF. Fisioterapia em grupo melhora o equilíbrio e a funcionalidade de indivíduos com hemiparesia. ConScientiae Saúde. 2016; 15(3):385-91.

5. Rothwell J, Burke D, Hicks R, Woodforth I, Crawford M. Transcranial electrical stimulation of the motor cortex in man: further evidence for the site of activation. J Physiol. 1994; 481(Pt 1):243-50.

6. Britto HMJS, Mendes LA, Moreno CC, Silva EMGS, Lindquist ARR. Correlation between balance, speed, and walking ability in individuals with chronic hemiparesis. Fisioter. mov. 2016;29(1):87-94.

7. Son SM, Park MK, Lee NK. Influence of Resistance Exercise Training to Strengthen 


\section{Fisi Y̌enenectus}

Bini AC, Gomes JC, Antonelli G, Dalzoto JS, Pereira GA

Muscles across Multiple Joints of the Lower Limbs on Dynamic Balance Functions of Stroke Patients. J Phys Ther Sci. 2014; 26(8): 1267-9.

8. Al-Jarrah M, Shaheen S, Harries N, Kissani N, Molteni F, Bar Haim S. MESF Project. Individualized treadmill and strength training for chronic stroke rehabilitation: effects of imbalance. Top Stroke Rehabil. 2014; 21 Suppl 1:525-32.

9. Lim HS, Kim YL, Lee SM. The effects of Pilates exercise training on static and dynamic balance in chronic stroke patients: a randomized controlled trial. J Phys Ther Sci. 2016; 28(6):1819-24.

10. Haruyama K, Kawakami M, Otsuka T. Effect of Core Stability Training on Trunk Function, Standing Balance, and Mobility in Stroke Patients: A Randomized Controlled Trial. Neurorehabil. Neural Repair. 2017; 31(3):240-9.

11. Kisner C, Thorp JN. The spine: management guidelines. In: Kisner C, Colby LA, eds. Therapeutic Exercise: Foundations and Techniques. 5th ed. Philadelphia; 2007.

12. Dobkin BH. "Strategies for stroke rehabilitation". Lancet Neurol. 2004; 3(9):528-36.
13. Silva WHS, Lopes GLB, Yano KM, Tavares NSA, Rego IAO, Cavalcanti FAC. Effect of a rehabilitation program using virtual reality for balance and functionality of chronic stroke patients. Motriz: rev. educ. fis. 2015; 21(3):237-43.

14. Choi HS, Shin WS, Bang DH, Choi SJ. Effects of Game-Based Constraint-Induced Movement Therapy on Balance in Patients with Stroke: A Single-Blind Randomized Controlled Trial. Am J Phys Med Rehabil. 2017; 96(3):184-90.

15. Costa GC, Corrêa JCF, Silva SM, Corrêa FI. Efeito do enfaixamento em oito no equilíbrio estático e distribuição de pressão plantar após acidente vascular encefálico. Fisioter. Pesqui. 2015; 22(4):398-403.

16. Filho EMS, Albuquerque JA. Influência da terapia de restrição e indução do movimento no desempenho funcional de pacientes com acidente vascular encefálico: um ensaio clínico randomizado. Fisioter. Pesqui. 2017; 24(2):184-90.

17. Park J, Seo D, Choi W, Lee S. The Effects of Exercise with TENS on Spasticity, Balance, and Gait in Patients with Chronic Stroke: A Randomized Controlled Trial. Med Sci Monit. 2014; 20:1890-6. 


\section{Anexos}

Fluxograma 1 - Descrição dos métodos para seleção de artigos.

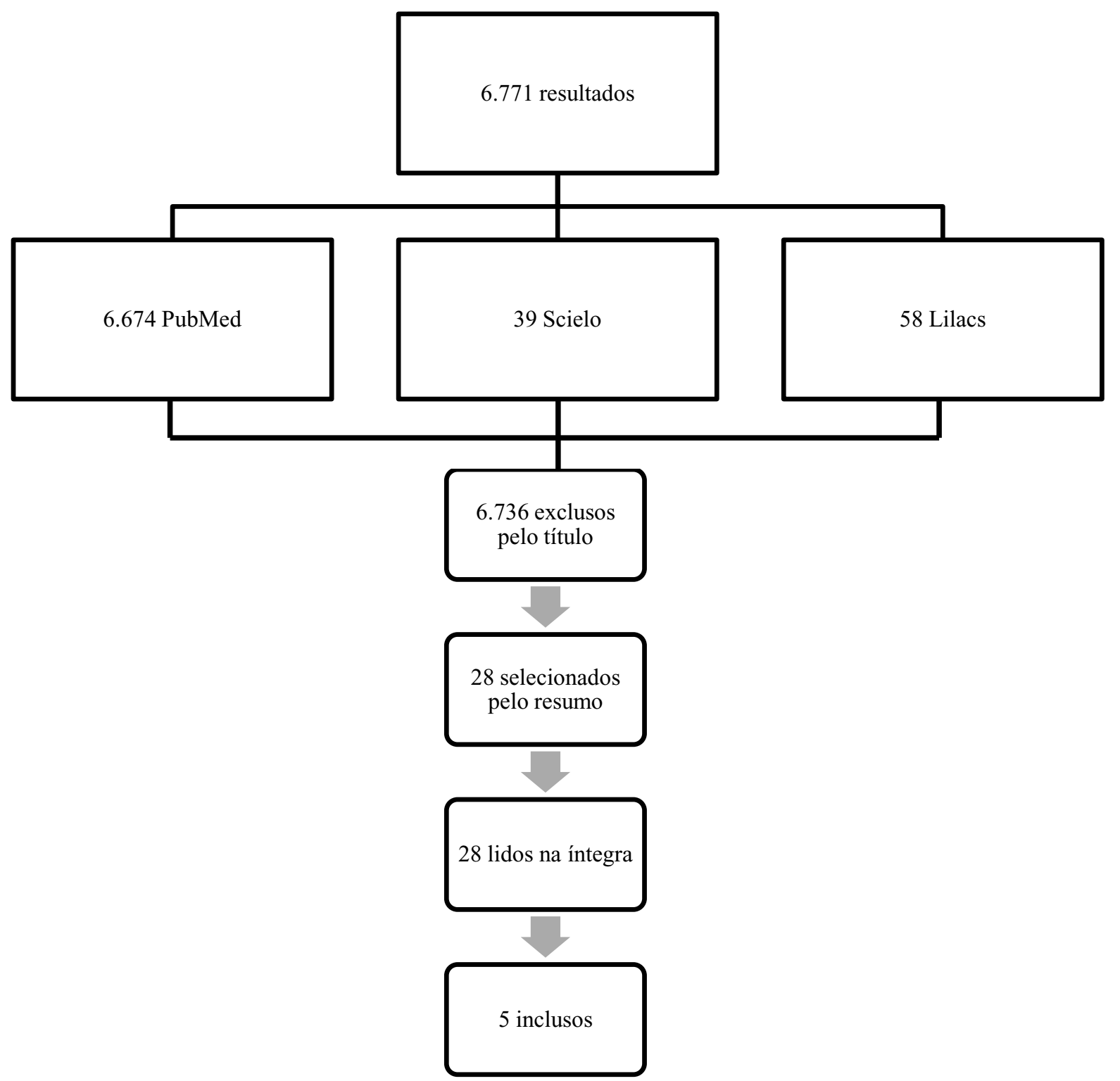

(clique para voltar ao texto) 


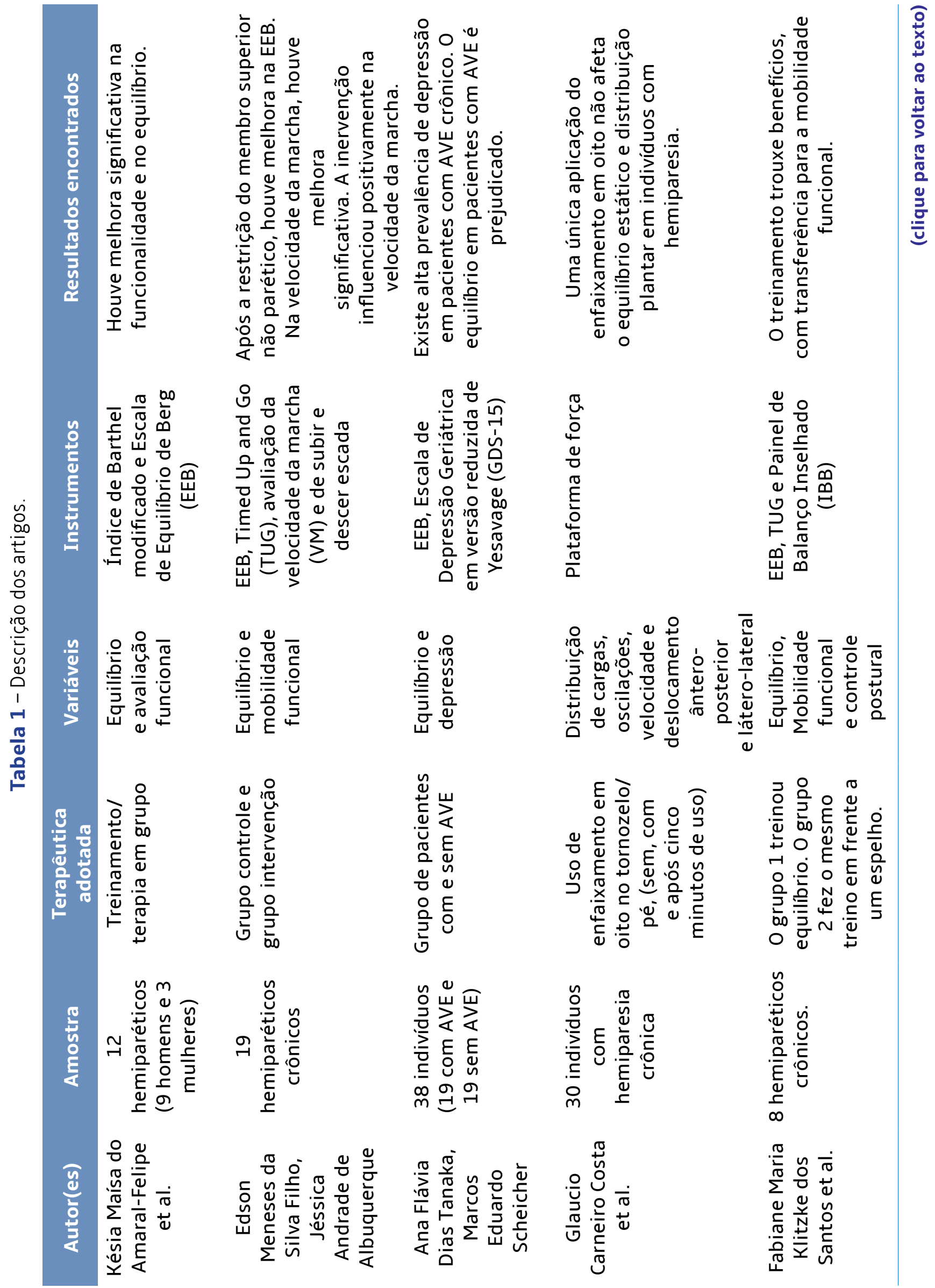

